\title{
Spherulitic Growth of Poly(ethylene oxide) from Viscous Solution
}

\author{
Takashi SASAKI, $^{\dagger}$ Yasuhisa Yamamoto, and Toshisada TAKAHASHI \\ Department of Materials Science \& Engineering, Fukui University, \\ 3-9-1 Bunkyo, Fukui 910-8507, Japan \\ (Received January 7, 1998)
}

\begin{abstract}
Spherulitic growth of poly(ethylene oxide) from $30 \mathrm{wt} \%$ tripropionin (glycerol tri- $n$-propionate) solution was investigated in the supercooling range $\Delta T=11-37 \mathrm{~K}$. The solution was pre-annealed at $200^{\circ} \mathrm{C}$ before isothermal crystallization. The estimated total crystallization rate decreases with increasing pre-annealing time, and becomes almost constant with a pre-annealing time longer than $c a .200 \mathrm{~h}$. This suggests that traces of crystalline nucleus remaining in the solution dissolve during the pre-annealing process. The isothermal crystallization rate $G$ with respect to the crystallization temperature was measured using isothermal calorimetric measurements and direct observation of the spherulitic growth using a CCD camera. The Avrami exponent estimated from the isothermal crystallization data ranges from $c a .2 .7$ to 3.2 depending on the crystallization temperature. The profile of $G$ vs. $T$ was also analyzed to estimate the parameters for the diffusion factor, and the result shows that the diffusion process belongs to the low friction limit, i.e., frictional interaction between the polymer and solvent is extremely weak.

KEY WORDS Poly(ethylene oxide) / Spherulite / Crystallization from Solution / Primary Nucleation / Activation Energy / Polymer-Solvent Interaction / Low Friction Limit /
\end{abstract}

The crystallization phenomenon of polymers has attracted much interest since the establishment of polymer science, but has not yet been fully understood in spite of a number of studies on the subject. The polymer crystallization rate is governed by thermodynamic driving force (entropy factor) and mobility of the polymer chain (diffusion factor). ${ }^{1,2}$ The melt (or bulk) crystallization has often been interpreted on the basic assumption that the diffusion factor can be expressed by the same formula that has been established for the polymer dynamics in the equilibrium melt state, e.g., the WLF expression or the reptation model. ${ }^{1,3-6}$ However, the polymer crystallization is essentially a non-equilibrium transport phenomenon, and at least, the diffusion models for the equilibrium system should not be uncritically applied to the polymer crystallization process.

In the case of polymer crystallization from solutions (especially from dilute solutions), the diffusion factor is often neglected especially at low supercooling because of weaker contribution than that of the entropy factor. However, diffusion during the solution crystallization is still an important and interesting problem because the anomalous feature of the polymer-solvent interaction in a non-equilibrium state is expected to play an important role. We found characteristic singularity in the polymersolvent interaction during solution crystallization at a certain higher concentration where the diffusion process cannot be neglected. ${ }^{7}$

In our previous study on solution crystallization of isotactic polystyrene, ${ }^{7}$ we evaluated the frictional interaction between polymer and solvent on the basis of the reaction rate theory of Kramers, ${ }^{8,9}$ and found that it is fairly reduced and strongly dependent on solvent. This may be explained by the idea that in the supercooled solution, where the polymer is essentially insoluble in the solvent, the solvent molecule has little tendency to be attached to the polymer chain, resulting in a small frictional polymer-solvent interaction. In addition, un-

\footnotetext{
${ }^{\dagger}$ To whom correspondence should be addressed.
}

realistic negative values of the activation energy of local polymer motions have been reported in highly viscous solvents even in equilibrium solutions, and this can also be interpreted as an anomalous low frictional polymer-solvent interaction..$^{10-12}$ Hence, it is expected that the feature of the low friction during the solution crystallization between polymer and solvent is more likely to be observed in a highly viscous medium.

This study investigates the isothermal crystallization of poly(ethylene oxide) (PEO) from a viscous solvent, tripropionin (glycerol tri- $n$-propionate, referred to as TP henceforth) by both the thermal analysis and the direct measurements of the spherulitic linear growth rate. As for the solution crystallization of PEO, kinetic data have been extensively collected for very dilute toluene solutions ${ }^{13,14}$ in which the contribution of diffusion is relatively small. The present study uses solution of higher concentration $(30 \mathrm{wt} \%)$, at which the diffusion factor plays an apparent role, and also spherulites of appropriate sizes can readily be obtained. Sufficiently large exothermic peaks concerning the solution crystallization can easily be observed by the DSC apparatus for the present concentrated system. From data on the crystallization rate with respect to the crystallization temperature (supercooling), we discuss PEO-TP interactions during solution crystallization.

\section{EXPERIMENTAL}

Commercial PEO $\left(M_{n}=2.85 \times 10^{4}, M_{w}=3.15 \times 10^{4}\right.$, $\left.M_{w} / M_{n}=1.11\right)$ was obtained from Wako Pure Chemical Industries. TP (Tokyo Kasei Co.) was distilled under vacuum and filtered using a $0.2 \mu \mathrm{m}$ mesh Millipore filter just before use. In a dust-free atmosphere (class 100), PEO was added to TP to make a $30 \mathrm{wt} \%$ solution. This mixture was then degassed under high vacuum $\left(<10^{-5}\right.$ $\mathrm{mmHg}$ ), and the pre-annealing for dissolution was executed at $200^{\circ} \mathrm{C}$ for up to $420 \mathrm{~h}$. Solution viscosity and FT-IR spectra of PEO (by a Nicolet Magna 560) were measured before and after pre-annealing to de- 
termine whether or not the degradation of PEO or any other reaction during the long time heat treatment occurs.

Isothermal measurement for crystallization was done for the solution pre-annealed at $200^{\circ} \mathrm{C}$ for $52 \mathrm{~h}$ by a DSC apparatus (Perkin Elmer DSC7) in aluminum sealed pans. The temperature readings of the apparatus were calibrated with an indium standard. In this study, we did not follow any intended seeding procedure: the $200^{\circ} \mathrm{C}$ pre-annealed solution was quenched to $90^{\circ} \mathrm{C}$, and then, was immediately put into an aluminum pan, being quenched to room temperature in the air $\left(c a .25^{\circ} \mathrm{C}\right)$. After the aluminum pan was sealed at room temperature, measurement of the isotherms was done with further annealing at $93^{\circ} \mathrm{C}$ for $10 \mathrm{~min}$ followed by quenching to various crystallization temperatures $\left(10-30^{\circ} \mathrm{C}\right)$.

We directly measured the spherulitic growth rate during isothermal crystallization from the solution preannealed at $200^{\circ} \mathrm{C}$ for $202 \mathrm{~h}$ by monitoring the solution using a CCD camera (Flovel HCC-600) equipped with a zoom lens (Navitar Macro Zoom). Temperature was controlled within $\pm 0.02^{\circ} \mathrm{C}$ by a thermo-regulated water bath with a Taitec DG- 10 . The $200^{\circ} \mathrm{C}$ pre-annealed solution was quenched to room temperature $\left(\mathrm{ca} .25^{\circ} \mathrm{C}\right)$ and held for more than one day. The isothermal spherulitic growth was observed after further annealing at $70^{\circ} \mathrm{C}$ for $8 \mathrm{~min}$ followed by quenching to the various crystallization temperatures $\left(10-35^{\circ} \mathrm{C}\right)$. All the above procedures were done under vacuum (in the degassed ampoule prepared before the pre-annealing). Image data were recorded by a videotape recorder, and sizes of the spherulites at various times were measured on a video monitor (Sony PVM-14550M).

To determine the equilibrium dissolution temperature, $T_{\mathrm{d}}^{\circ}$, a $30 \mathrm{wt} \%$ PEO-TP solution was crystallized in the sealed aluminum pan at various temperatures in a Perkin Elmer DSC7, and the dissolution temperature was measured by a successive heating scan at the rate of $10^{\circ} \mathrm{Cmin}^{-1}$. By the extrapolation method, ${ }^{15}$ we determined $T_{\mathrm{d}}{ }^{\circ}$ for the present system to be $46.4^{\circ} \mathrm{C}$. In general, the equilibrium temperature estimated by the present extrapolation method tends to be low with some uncertainty compared with that from the method based on the direct measurement of lamellar thickness (GibbsThomson formula). However, for $T_{\mathrm{d}}{ }^{\circ}$ of higher molecular weight PEOs, the latter method does not necessarily give a more accurate value than the present method. ${ }^{14}$ The viscosity of TP was measured at various temperatures using an Ubbelohde type viscometer, and the activation energy of the viscosity was determined to be $6.50 \mathrm{kcal}$ $\mathrm{mol}^{-1}$.

\section{RESULTS AND DISCUSSION}

The viscosity of the PEO-TP solution was reduced by $0.8 \%$ after the annealing at $200^{\circ} \mathrm{C}$ for $202 \mathrm{~h}$ under vacuum. We recognized no apparent change in the FTIR spectrum after the pre-annealing. PEO spherulites developed at $10-35^{\circ} \mathrm{C}$ in the present TP solution of rather high concentration $(30 \mathrm{wt} \%)$ in spite of no intended seeding procedure. Figure 1 shows examples of growing spherulites. Considerable change in the time needed for the spherulitic growth of PEO with respect to the pre-annealing time was noted. For isothermal (a)

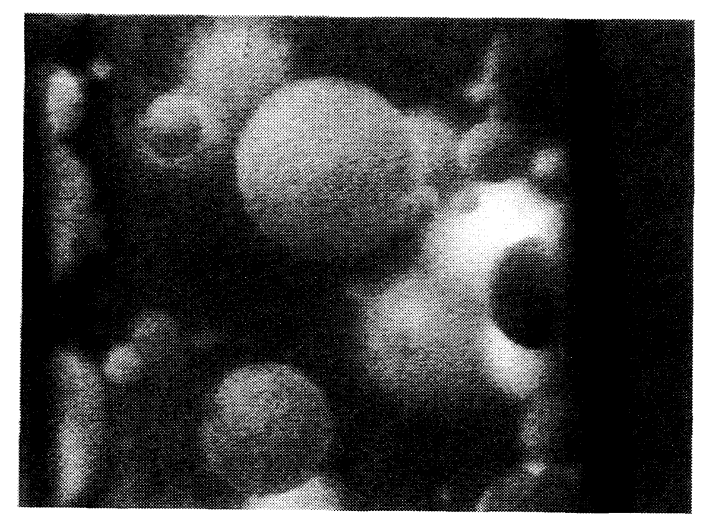

(b)

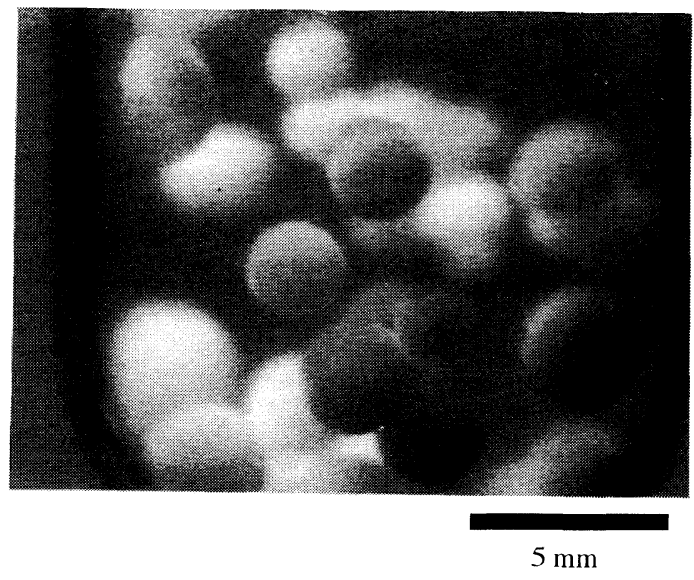

Figure 1. Growing PEO spherulites in $30 \mathrm{wt} \% \mathrm{TP}$ solution at (a) $16^{\circ} \mathrm{C}$ and (b) $33^{\circ} \mathrm{C}$.

crystallization at $30^{\circ} \mathrm{C}(\Delta T=16.4 \mathrm{~K})$, we measured the time when the first visible spherulite appears in the solution $\left(T_{\mathrm{i}}\right)$, and the time when the entire solution becomes filled with spherulitic crystals $\left(T_{\mathrm{f}}\right) . T_{\mathrm{i}}$ is a rough measure of the induction period for the crystallization, and $T_{\mathrm{f}}$ is a rough estimate for the total crystallization time (inversely related to the total crystallization rate). Here, for convenience, $T_{\mathrm{i}}$ and $T_{\mathrm{f}}$ were determined by measuring the time when the foremost spherulite of recognizable size with the naked eye $\left(c a \cdot 10^{-1} \mathrm{~mm}\right.$ for diameter) appears in the solution, and by measuring the time when a dry area first appears anywhere at the surface of the glass tube where the solvent is soaked up into the spherulite. When the initial spherulite was detected at $T_{\mathrm{i}}$, it was too small to scrutinize its morphology, but it grew into a spherical particle (see Figure 1).

Figure 2 shows the above two characteristic times with respect to annealing time. The data show that both $T_{\mathrm{i}}$ and $T_{\mathrm{f}}$ increase with the annealing time. Such behavior of $T_{\mathrm{f}}$ indicates that total crystallization rate decreases with increasing pre-annealing time. The tendency seems weaker with increasing annealing time, and $T_{\mathrm{i}}$ and $T_{\mathrm{f}}$ become almost constant after $c a .200 \mathrm{~h}$.

The results in Figure 2 may be partially due to the degradation of PEO (hydrolysis or thermal degradation) during the annealing: the reduction of molecular weight by the degradation causes a lower equilibrium dissolution temperature and lower supercooling, and as a result, the crystallization rate decreases. However, considering the present small change in viscosity and no apparent change in the FT-IR spectra, we attribute the above results mainly to the change in the inhomogeniety of the PEO- 


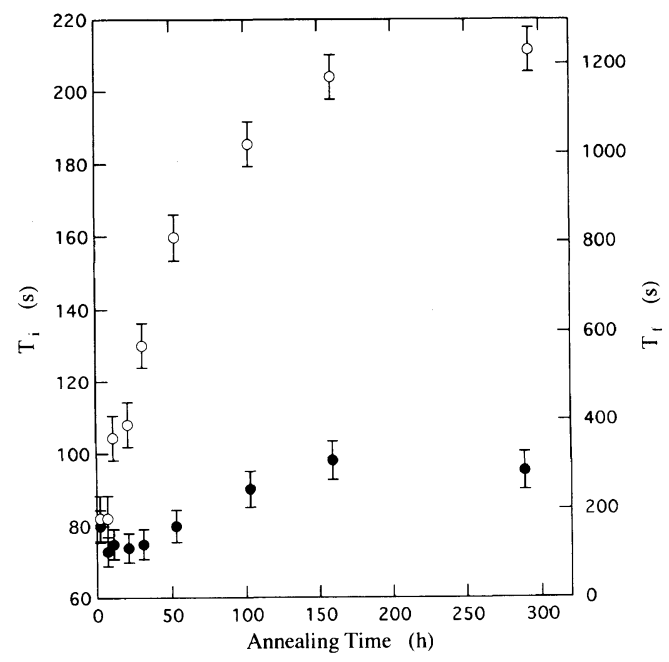

Figure 2. Two characteristic times, $T_{\mathrm{i}}(\bigcirc)$ and $T_{\mathrm{f}}(\bigcirc)$ (see the text), with respect to pre-annealing time.

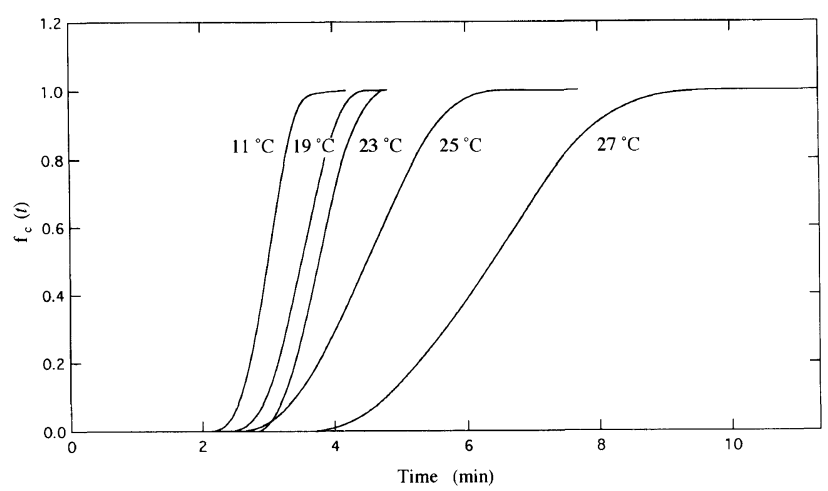

Figure 3. Time evolution of the extent of crystallization, $f_{c}(t)$, for various crystallization temperatures obtained from the isothermal calorimetry by the DSC apparatus.

TP solution during the pre-annealing. When annealing is not enough, residuals (traces) of the crystalline, or small regions in which the PEO chains take up a crystalline-like conformation still remain in the solution, and they act as seeds for the primary nucleation when the solution is quenched to crystallization temperatures. These inhomogeneous residual sites in the solution gradually dissolve as the pre-annealing proceeds above the dissolution temperature. As a result, the number of primary nucleation sites in the solution decreases, and the total crystallization rate decreases resulting in enhancement of $T_{\mathrm{f}}$. Also, the behavior of $T_{\mathrm{i}}$ (the induction period) in Figure 1 may be due to the above reduction of nucleation sites.

It may be surprising that a significantly severe annealing $\left(200^{\circ} \mathrm{C}\right.$ for $\left.200 \mathrm{~h}\right)$ is needed to obtain an almost homogeneous solution. This means that the structural relaxation time for such inhomogeneous structure of the solution is much longer than the crystallization time of the present experiments (less than ca. $30 \mathrm{~min}$ ), and heterogeneous nucleation is supposed to be dominant for the present system.

We noted that the total number of spherulites that finally appeared in the solution during the crystallization (from $T_{\mathrm{i}}$ to $T_{\mathrm{f}}$ ) decreases with increasing annealing time. The final number density of the spherulite appeared at $30^{\circ} \mathrm{C}$ in the solution without the pre-annealing was too

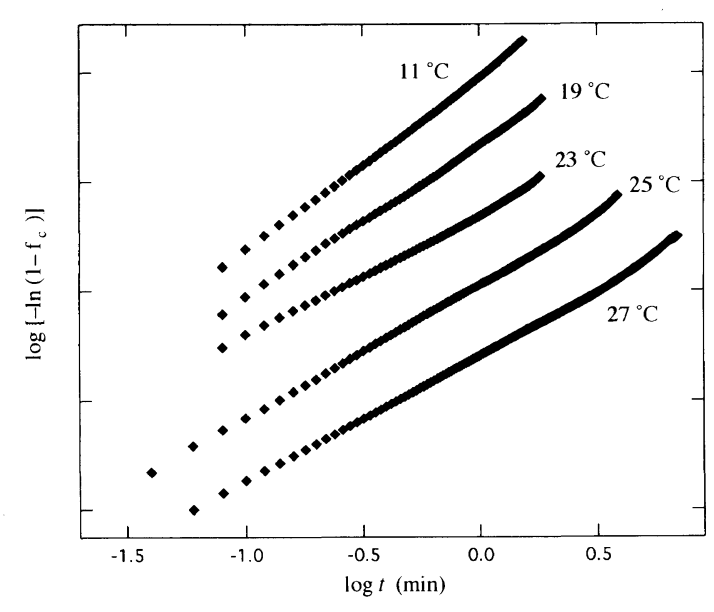

Figure 4. Avrami plots for the data in Figure 2.

large $\left(>50 \mathrm{~g}^{-1}\right)$ to estimate quantitatively by the present method, but it decreased to $c a$. $7-9 \mathrm{~g}^{-1}$ after the annealing for $202 \mathrm{~h}$ at $200^{\circ} \mathrm{C}$. Though we could not determine directly the primary nucleation rate (the frequency of nucleation per unit time) from the present experiment, the obtained result may suggest that the primary nucleation rate is reduced by the pre-annealing, and this is consistent with the above consideration that the primary nucleation sites (the crystalline residual sites) decrease during pre-annealing above the dissolution temperature.

Figure 3 shows the extent of crystallization $f_{\mathrm{c}}(t)$ at various crystallization temperatures, which was obtained from the isothermal calorimetric data according to

$$
f_{\mathrm{c}}(t)=\int_{0}^{t} H\left(t^{\prime}\right) \mathrm{d} t^{\prime} / \int_{0}^{\infty} H\left(t^{\prime}\right) \mathrm{d} t^{\prime}
$$

where $H\left(t^{\prime}\right)$ is the excess heat flow for the exothermic crystallization peak. Figure 4 shows the corresponding Avrami plots for the data in Figure 3. By introducing an appropriate induction period, we get almost linear profiles in the Avrami plots for all the obtained data. This indicates that the type of spherulitic growth process does not change throughout the entire crystallization process in the aluminum pan, and in addition, that the decrease in the concentration of the remaining PEO solution during the isothermal crystallization does not apparently reduce the crystallization rate. From the present result, we consider that in the solution in which the spherulites are growing, the concentration of PEO in the liquid domain is not homogeneously distributed throughout the test tube; in other words, the local concentration of PEO is kinetically different between the inside and outside of the spherulitic particles.

Figure 5 shows the obtained Avrami exponent $n$ with respect to the crystallization temperature. We see that $n$ is almost constant $(c a .3 .2)$ below $20^{\circ} \mathrm{C}$, while it becomes lower (ca. 2.7) above $20^{\circ} \mathrm{C}$. This may be due to the fact that the probability of the primary nucleation decreases with decreasing supercooling. From the above estimated density of spherulite appeared in the solution $\left(7-9 \mathrm{~g}^{-1}\right.$ at $30^{\circ} \mathrm{C}$ ), we can say that at high crystallization temperatures, the mean number of the spherulites in an aluminum pan is less than 1 (the sample weight was $c a$. $20 \mathrm{mg}$ ). At temperatures above $20^{\circ} \mathrm{C}$, at most, a single 


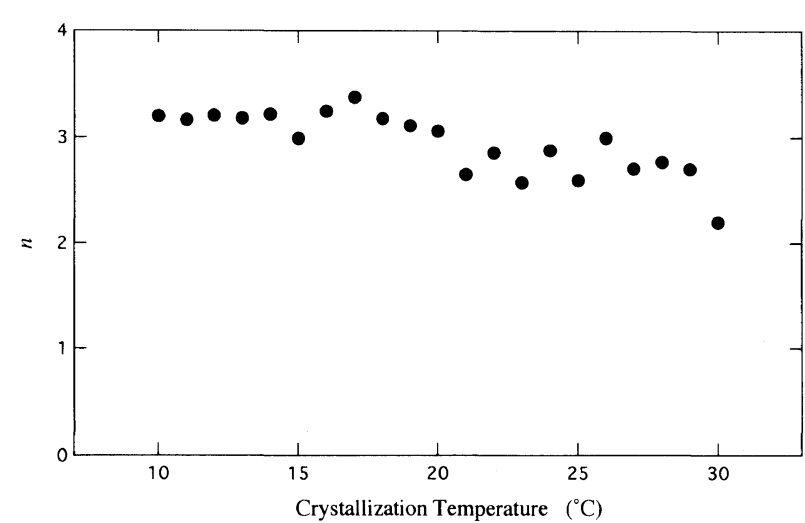

Figure 5. Avrami exponent $n$ of the isothermal spherulitic growth evaluated from the calorimetric data.

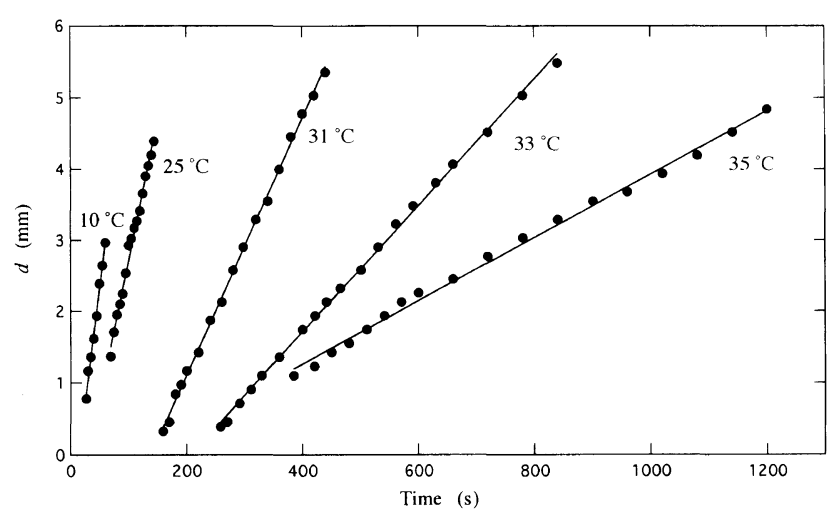

Figure 6. Diameter $d$ of the PEO spherulite during the isothermal crystallization from $30 \mathrm{wt} \%$ TP solution with respect to time at different crystallization temperatures.

spherulite may thus grow in a sample pan, leading to smaller $n$ value than that below $20^{\circ} \mathrm{C}$ where multiple spherulites develop.

The crystallinity of the obtained spherulitic materials was estimated from the area of the exothermic peak to be $59-66 \%$ with no apparent dependence on the crystallization temperature. The present crystallinity is fairly lower than that of the bulk crystallization, ${ }^{16}$ which indicates that solvent molecules hamper the formation of crystalline conformation of the PEO chain.

Figure 6 shows the observed diameters $d$ of the spherulites with respect to the crystallization time, indicating that the diameter increases linearly with time. We estimated the spherulitic growth rate $G_{\mathrm{CCD}}$ from the slopes of these lines as shown in Figure 7. Figure 7 shows the results from the thermal measurement by the DSC apparatus; the isothermal crystallization rate $G_{\mathrm{DSC}}$ was estimated with the reciprocal of the time for reaching the value 0.5 for the extent of crystallization $f_{c}(t)$. We see that the profiles of the $G v s .1 / T \Delta T$ data obtained from the two different methods are very similar to each other. According to the standard surface nucleation theory, ${ }^{2,17-19} \ln G$ should decrease linearly with $1 / T \Delta T$. The slight non-linearity of $\ln G$ vs. 1/T $T$ plot seen in Figure 7 suggests that at least one of the following three factors should be considered: (1) diffusion of the polymer chains (diffusion factor), (2) the regime transition, ${ }^{20}$ and (3) fractionation according to molecular weight. ${ }^{21-23}$ Since spherulitic growth is observed at all the supercoolings with no change in morphology, we do not

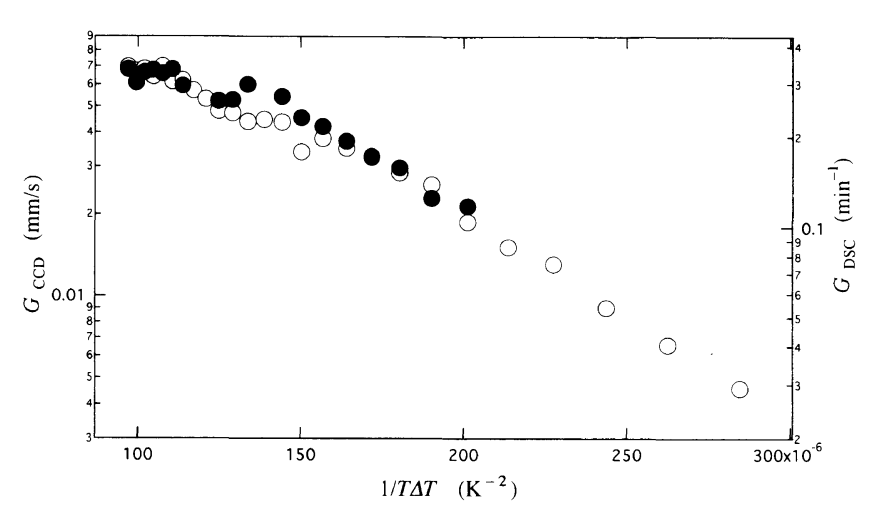

Figure 7. Isothermal crystallization rate $G$ with respect to $1 / T \Delta T$ evaluated from the direct measurements of the diameter of the spherulite $\left(G_{\mathrm{CCD}}, \bigcirc\right)$, and from the calorimetry $\left(G_{\mathrm{DSC}}, \bigcirc\right)$.

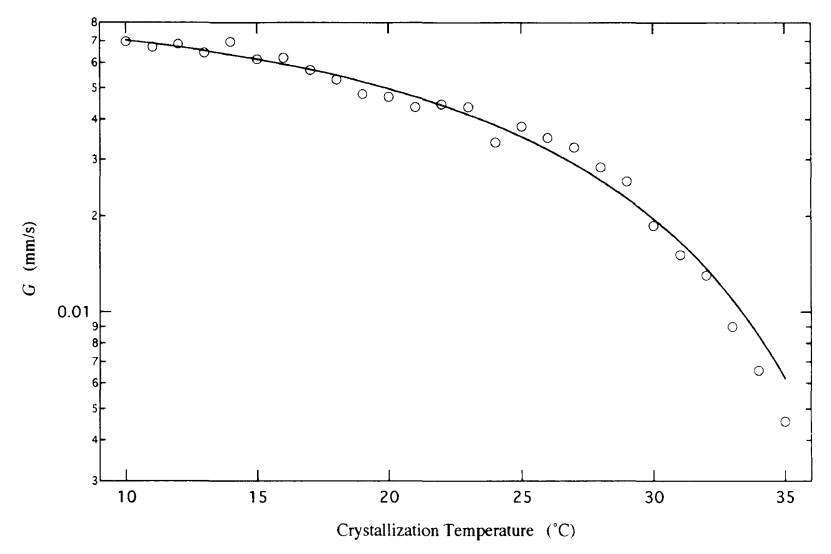

Figure 8. Isothermal crystallization rate $G$ obtained from the diameter measurement with respect to the crystallization temperature. The solid line indicates the fitted curve obtained by the analysis using eq 2.

presently consider the regime transition for the present system.

To take into account the diffusion factor, we analyzed the observed $G$ vs. $T$ data according to the following expression $^{7}$

$$
\begin{aligned}
G= & G_{0} \exp \left(-\frac{K_{\mathrm{g}} T_{\mathrm{d}}{ }^{\circ}}{T \Delta T}\right) \exp \left[-\left(E_{\mathrm{a}}+E_{\eta}\right) / R T\right] / \\
& \left\{0.5+\left[0.25+A_{1} \exp \left(-2 E_{\eta} / R T\right)\right]^{0.5}\right\}
\end{aligned}
$$

with

$$
K_{\mathrm{g}}=\frac{x b \sigma \sigma_{\mathrm{e}}}{k \Delta H}
$$

where $G_{0}$ is the pre-exponential factor, $E_{\mathrm{a}}$ and $E_{\eta}$ are the activation energy of the polymer diffusion and solvent viscosity, respectively, $A_{1}$ is a parameter determining the potential profile for the polymer chain diffusion, $x$ depends on the regime of surface nucleation ( 2 or 4$), b$ is the thickness of a stem, $\sigma$ is the lateral surface free energy, $\sigma_{\mathrm{e}}$ is the end surface free energy, and $\Delta H$ is the polymer heat of dissolution. Analysis was done by directly fitting the calculated curve based on eq 2 to the experimental data of $G_{\mathrm{CCD}}$ using the non-linear least squares fitting algorithm (modified Marquardt method).

Figure 8 shows the above fitted curve together with experimental data from the diameter measurements, and Table I gives the obtained parameters from the analysis. 
Table I. Data obtained from fitting analysis for the $G_{\mathrm{CCD}} v s . T$ data

\begin{tabular}{ccc}
\hline$E_{\mathrm{a}} / \mathrm{kcal} \mathrm{mol}^{-1}$ & $\ln A_{1}$ & $K_{\mathrm{g}} / \mathrm{K}$ \\
\hline 1.97 & 47.8 & 45.4 \\
\hline
\end{tabular}

We note in Table I that $A_{1}$ is extremely large $(5.74 \times$ $10^{20}$ ), which means that the diffusion factor can be practically expressed as $\exp \left(-E_{\mathrm{a}} / R T\right)$ (the low friction limit $\left.{ }^{8,9}\right)$. In other words, the solvent viscosity does not affect the polymer diffusion, and thus, frictional interaction between the polymer and solvent is extremely weak. This was expected for the present system, because PEO in a supercooled solution is essentially incompatible to the solvent, and in this case, PEO chains may crystallize slipping through the solvent molecules with low friction. We found the same low-frictional feature in the solution crystallization of the isotactic polystyrene-TP system, ${ }^{7}$ and we consider that such a low-friction aspect between the polymer and solvent is closely related to the specific character of TP itself. In general, the low polymer-solvent friction in a high viscosity medium can be interpreted as follows. Solvent molecules possessing ester groups like TP tend to associate with each other to form a network structure resulting in high viscosity, ${ }^{12}$ and the polymer molecules merely move through the rather set network with low friction. For the present system, the solvent network is probably well established in spite of the large content of PEO (30 wt \%) which may hinder network formation.

The obtained $E_{\mathrm{a}}$ is in the range evaluated from the conformational energy calculations for the PEO segmental chain, ${ }^{24}$ which may correspond to the activation energy for the local conformational transitions. We estimated $\sigma_{\mathrm{e}}$ to be $16.3 \mathrm{erg} \mathrm{cm}^{-2}$ using the literature values for the parameters $b=4.63 \AA,{ }^{25} \Delta H=2.31 \times 10^{9}$ $\mathrm{erg} \mathrm{cm}^{-3},{ }^{26}$ and $\sigma=9.6 \mathrm{erg} \mathrm{cm}^{-2},{ }^{27,28}$ and with the assumption that $x=2$ (regime II). The value obtained here is much smaller than in other studies on crystallization from dilute solutions. ${ }^{13,14}$ The reason for this is not clear, though there may be a problem in employing the above literature values obtained under different experimental conditions.

In Figure 8, we note systematic deviation at high crystallization temperatures $\left(>31^{\circ} \mathrm{C}\right)$, where experimental values are lower than the theoretical curve. This may indicate that the fractionation effect according to molecular weight exists. ${ }^{21-23}$ Such fractionation is due to different $T_{\mathrm{d}}$ 's for different molecular weight fractions, and thus to different crystallization rates with respect to molecular weight. In this case, the crystallization rate decreases with decreasing molecular weight, and lower $G$ of the experimental data at high crystallization temperatures may be due to the low molecular weight fractions contained in the present sample of which the crystallization rates are extremely low. We have no clear evidence for the formation of any integral folded (IF) or extended chain crystals ${ }^{29}$ in the present study, but the transition between the possible IF lamellar structures of different fold numbers for the low molecular weight fractions may also affect the present $G$ vs. $T$ data.

\section{CONCLUSIONS}

In the present study, we examined the crystallization of PEO from a viscous TP solution of $30 \mathrm{wt} \%$, and spherulitic growth was found to occur. The polymersolvent frictional interaction in the present system is extremely weak (the low friction limit holds), i.e., the PEO molecules move independent of the surrounding molecules during the spherulitic growth. This may be consistent with the idea that in the supercooled solution, the polymer chains that are essentially incompatible with the solvent have a weak interaction of friction with the solvent molecules. At higher polymer concentrations, a fairly large portion of the surrounding molecules must be occupied by PEO itself, and as a result, the frictional interaction between polymer and the surrounding molecules must become strong. Nevertheless, the anomalous low-frictional feature is still observed for the present concentrated solution ( $30 \mathrm{wt} \%)$, and we feel much excited about this result.

Deviation between the theory and experiment at high crystallization temperatures seen in Figure 8 may indicate the occurrence of fractionation according to molecular weight. Further investigation should be done on PEO fractions with extremely narrow molecular weight distributions to scrutinize this phenomenon and the corresponding variation in the chain folding feature.

Acknowledgment. This work was supported by a Grant-in-Aid for Scientific Research (No. 08751042) from the Ministry of Education, Science, Sports and Culture of Japan.

\section{REFERENCES}

1. D. Turnbull and J. C. Fisher, J. Chem. Phys., 17, 71 (1949).

2. K. Armistead and G. Goldbeck-Wood, Adv. Polym. Sci., 100, 219 (1992).

3. J. H. Magill, J. Appl. Phys., 35, 3249 (1964).

4. D. Turnbull, J. Appl. Phys., 21, 1022 (1950).

5. E. A. DiMarzio, C. M. Guttman, and J. D. Hoffman, Faraday Discuss. Chem. Soc., 68, 210 (1979).

6. J. D. Hoffman, Polymer, 23, 656 (1982).

7. T. Sasaki, M. Kurita, T. Yabu, and T. Takahashi, Macromolecules, 28, 8528 (1995).

8. H. A. Kramers, Physica, 7, 284 (1940).

9. E. Helfand, J. Chem. Phys., 54, 4651 (1971).

10. S. Yokotsuka, Y. Okada, Y. Tojo, T. Sasaki, and M. Yamamoto, Polym. J., 23, 95 (1991)

11. D. B. Adolf, M. D. Ediger, T. Kitano, and K. Ito, Macromolecules, 25, 867 (1992).

12. K. Ono, Y. Okada, S. Yokotsuka, S. Ito, and M. Yamamoto, Polym. J., 26, 199 (1994).

13. N. Ding, E. J. Amis, M. Yang, and R. Salovey, Polymer, 29, 2121 (1988).

14. N. Ding and E. J. Amis, Macromolecules, 24, 3906, 6464 (1991).

15. J. D. Hoffman and J. Weeks, J. Chem. Phys., 37, 1723 (1962).

16. S. Z. D. Cheng, J. Chen, J. S. Barley, A. Zhang, A. Habenschuss, and P. R. Zschack, Macromolecules, 25, 1453 (1992).

17. J. D. Hoffman and J. I. Lauritzen, J. Res. Natl. Bur. Stands., 65A, 297 (1961).

18. J. D. Hoffman and J. I. Lauritzen, J. Appl. Phys., 44, 4340 (1973).

19. J. I. Lauritzen, J. Appl. Phys., 44, 4353 (1973).

20. J. D. Hoffman, L. J. Frolen, G. S. Ross, and J. I. Lauritzen, J. Res. Natl. Bur. Stands., 79A, 671 (1975).

21. J.-J. Point and J. J. Janimak, J. Cryst. Growth, 131, 501 (1993).

22. J.-J. Point, J. Chem. Soc., Faraday Trans., 91, 2565 (1995). 
23. J.-J. Point, Macromolecules, 30, 1375 (1997).

24. I. Bahar, B. Erman, and L. Monnerie, Macromolecules, 22, 2396 (1989), and papers cited therein.

25. Y. Takahashi and H. Tadokoro, Macromolecules, 6, 672 (1973).

26. M. Pracella, Eur. Polym. J., 21, 551 (1985).
27. D. G. Thomas and L. A. K. Slaveley, J. Chem. Soc., 4569 (1952).

28. Y. Nishio, N. Hirose, and T. Takahashi, Sen'i Gakkaishi, 46, 441 (1990).

29. S. Z. D. Cheng, A. Zhang, J. Chen, and D. P. Hebberer, J. Polym. Sci., Polym. Phys. Ed., 29, 287 (1991), and succeeding papers. 